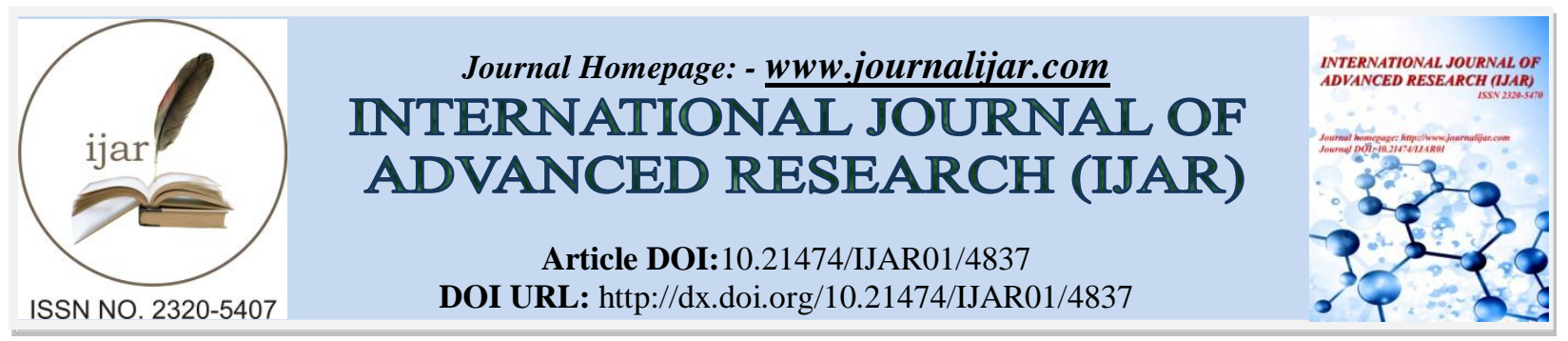

RESEARCH ARTICLE

\title{
COLLAPSED CHROMATIN DETECTION AND STRUCTURAL ABERRATIONS DETECTION BY STAINING.
}

Eswari Beeram, Kamala Katepogu and Divya Bysani.

$\mathrm{PhD}$, department of biochemistry, srivenkateswara university, tirupati-517502, Andhrapradesh.

\section{Manuscript Info}

Manuscript History

Received: 13 May 2017

Final Accepted: 15 June 2017

Published: July 2017

Key words:-

Collapsed chromatin, Condensed

chromatin, RNase protection,

nucleosomes, $30 \mathrm{~nm}$ fiber.

\section{Abstract}

Chromatin condensation involves folding to highly ordered structures which can be assembled and disassembled during gene expression. Aniline blue staining was performed in both fresh and frozen tissues of testis during which RNase protection of collapsed chromatin was observed, and collapsed chromatin was mainly due to apoptosis, that is known from the previous records. .

\section{Introduction:-}

Cell nucleus is a highly organized structure which is compartmentalized as membranes in the cell. Cell chromatin is organized as heterochromatin and euchromatin, in which heterochromatin includes transcriptionally inactive regions where as euchromatin represents the actively transcribed and replicated regions. Chromatin condensation involves folding to higher order structures like $30 \mathrm{~nm}$ fiber, solenoid model which are next to nucleosome. Certain histone modifications like $\mathrm{H} 1$ and $\mathrm{H} 3$ phosphorylation that occurs during mitotic condensation of chromosomes allows individualization of chromosomes. (3) This mitotic condensation reduces the interacting structures on the chromatin otherwise which was easily accessible. It is however not clear that how these chromatin sub structures allow accessibility to various nuclear proteins and chromatin organizing proteins. It is therefore clearly understood that RNA and protein synthesis is mainly responsible for the maintenance of chromatin architecture, inhibition of which leads to aggregation of chromatin, which was the reason for formation of massive clumps ${ }^{(6)}$. RNase is normally distributed in the endothelium ${ }^{(4)}$ lining heart valves, lymphatic vessels and blood vessels. There were reports showing its distribution in the testicular cells also ${ }^{(1)}$. Chromatin mainly collapses during apoptosis which occurs during certain regulated processes such as senescence, when the cells are about to die. Apoptosis is a required process that occurs during embryogenesis, cancer and during removal of unwanted cells in system. The main mediators of apoptosis are caspases that belongs to the family of cysteine and aspartic proteases those are synthesized as zymogens in healthy cells and activated by the cleavage process, for example TNF pathway. $\mathrm{H} 2 \mathrm{O} 2$ is one of the main messengers that are involved in apoptosis of sperm cells whose mechanism is largely unknown ${ }^{(2)}$. Previous reports have revealed that spontaneous degeneration, various stages of development and various degeneration pathways that occur in spermatogonia leads to depletion o f $70 \%$ of the mature sperms. It was known that plasma membrane consists of polyunsaturated fatty acids which can be readily prone to oxidation by ROS majorly $\mathrm{H} 2 \mathrm{O} 2$. 


\section{Results:-}

From the figure 1 it was clearly known that it was collapsed chromatin by the comparison of the two figures. It simply explains that collapsed chromatin or disassembly occurs mainly during apoptosis. It was clearly indicated that the chromatin collapse can be detected by simple aniline blue staining... From Figure 2 it was clearly known that due to RNase protection the condensation of chromatin was maintained as such which has to be proved further by other methods. From the figure 3(a), (b), (c), (d) it was clearly shown that it is highly compacted structure which is the solenoid model formed due to compaction of nucleosomes.

The arrows clearly show the nucleosome compaction. The images were obtained after 10 days of slide preparation. Aniline blue stain was taken densely in some regions of the tissue and other regions have taken the less stain compared to the dense regions which tells that it is less mature than the dense tissue.

Acridine orange results have showed that chromosomes are abberrated like anything. In control chromosomes are normal but there is micronuclei formation. In RNase treated one the sperm chromatin has bifurcated and also resulted in cyst formation. In drug treated one the heads of sperm has separated and hyaluridin in membrane are visible, tail has fragmented but the sperms are alive. In both RNase and drug treated one the chromosomes have reduced in size.

Agarose gel electrophoresis of treated samples has revealed that the sample was so fragmented and spread to all the wells

\section{Discussion:-}

Aniline blue stain is normally used to distinguish the mature and immature chromatin but in this paper is clearly shown to detect the collapsed chromatin which is due to apoptosis by simple staining procedure. Chromatin condensation in to highly compacted structures like solenoid model and 30nm fiber is clearly shown. RNase distribution is commonly seen in endothelium and reports have also shown it to be in testis also. Apoptosis is the one of the key feature of cells undergoing senescence. Caspases are mainly responsible for activation of apoptosis through two different pathways which is extrinsic and intrinsic. It is known to be that $\mathrm{H} 2 \mathrm{O} 2$ is the main mediator of apoptosis in testis. Seminiferous epithelium is mainly occupied by germ cells until they are developed in to matured sperms. In this article collapsed chromatin and RNase protection of collapse has been observed. It will be the great achievement to the patients if we develop the remedy for who is infertile due to chromatin collapse in testis due to diseases like cancer.

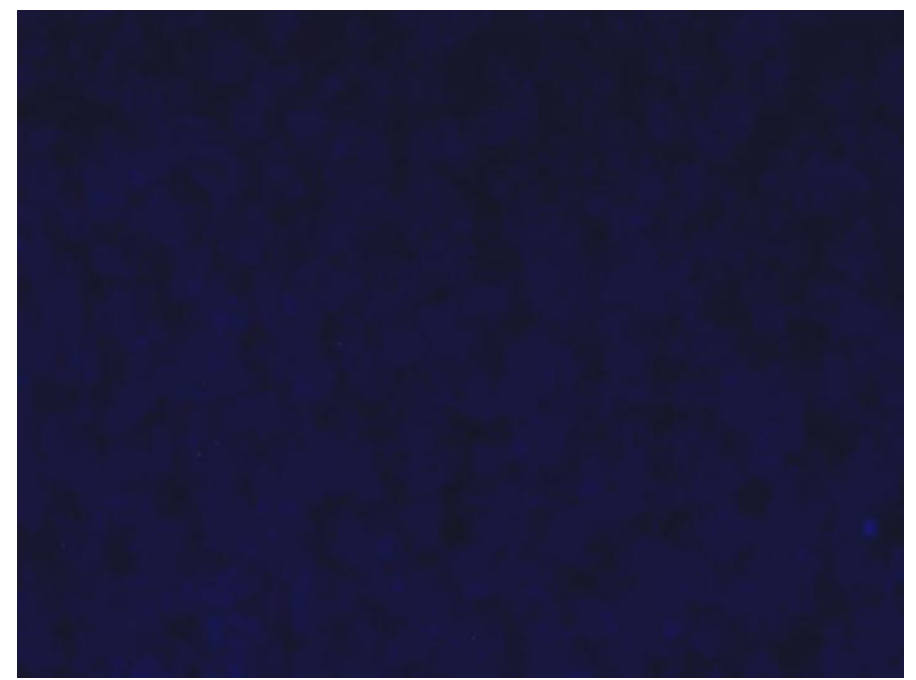

Figure 1:- It clearly shows that it is a collapsed chromatin which was stained with aniline blue after freezing the tissue nearly 1 month. 
Figure 2:- it was obtained from frozen tissue, but it clearly explains RNase expression in testis which can be explained by the chromatin organization.
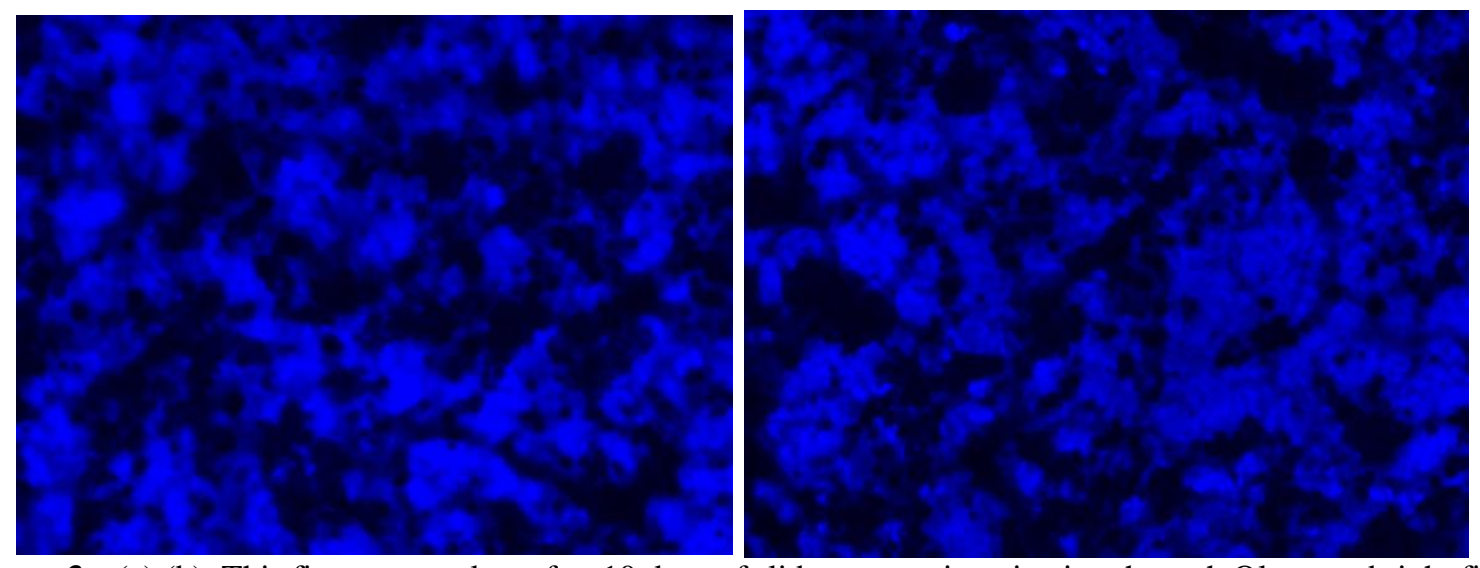

Figure 3:- (a),(b) This figure was taken after 10 days of slide preparation viewing through Olympus bright field microscope which is tissue taken from testis of nearly 3 months old. 


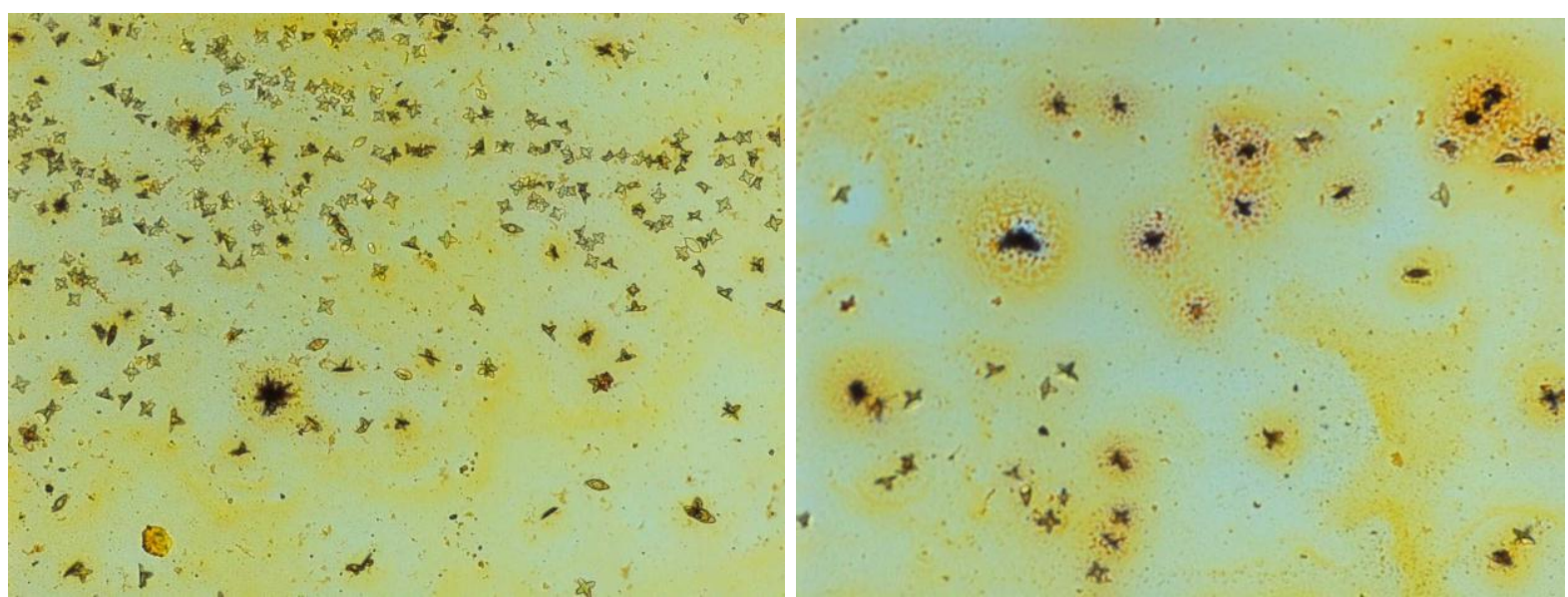

Figure 4:-acridine orange staining of control testis. Chromosomes are normal and micronuclei formation was seen.

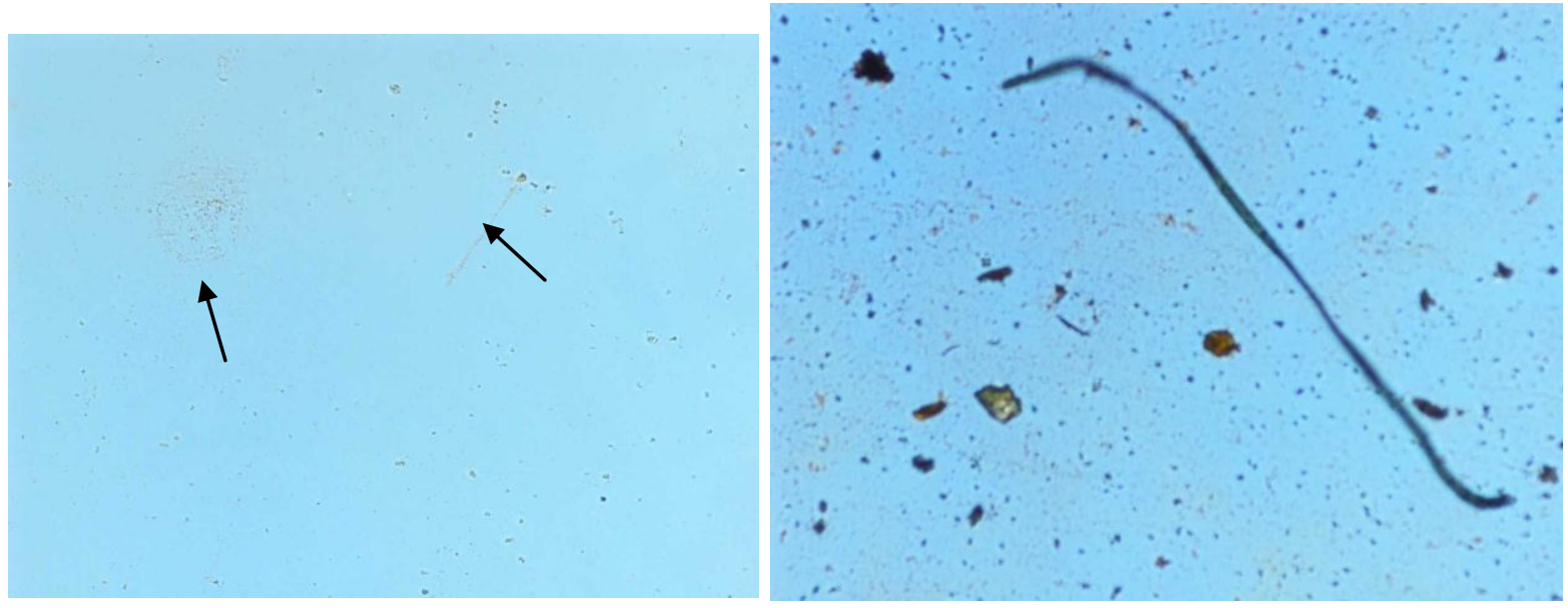

Figure 5:- testis treated with drug metosartan. Sperm was fragmented and head membrane was disintegrated.
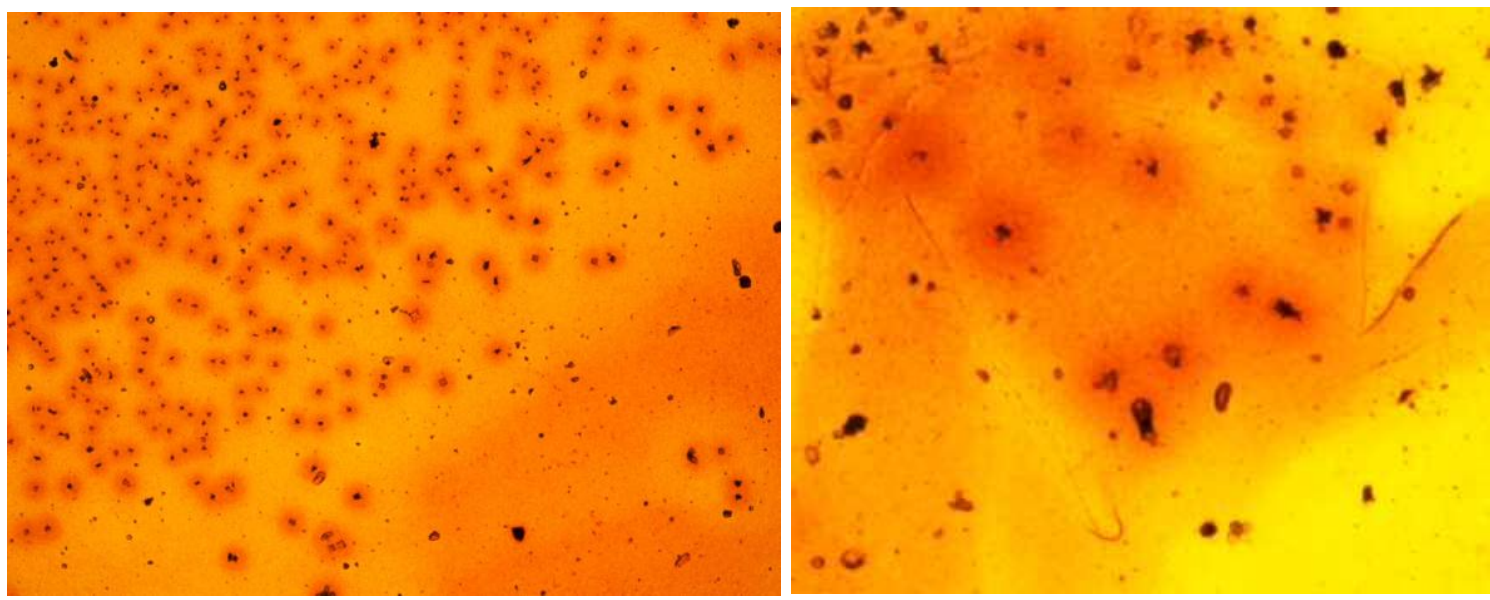

Figure 6:- testis treated with RNase A and drug metosartan: structural aberrations of chromosomes was seen 


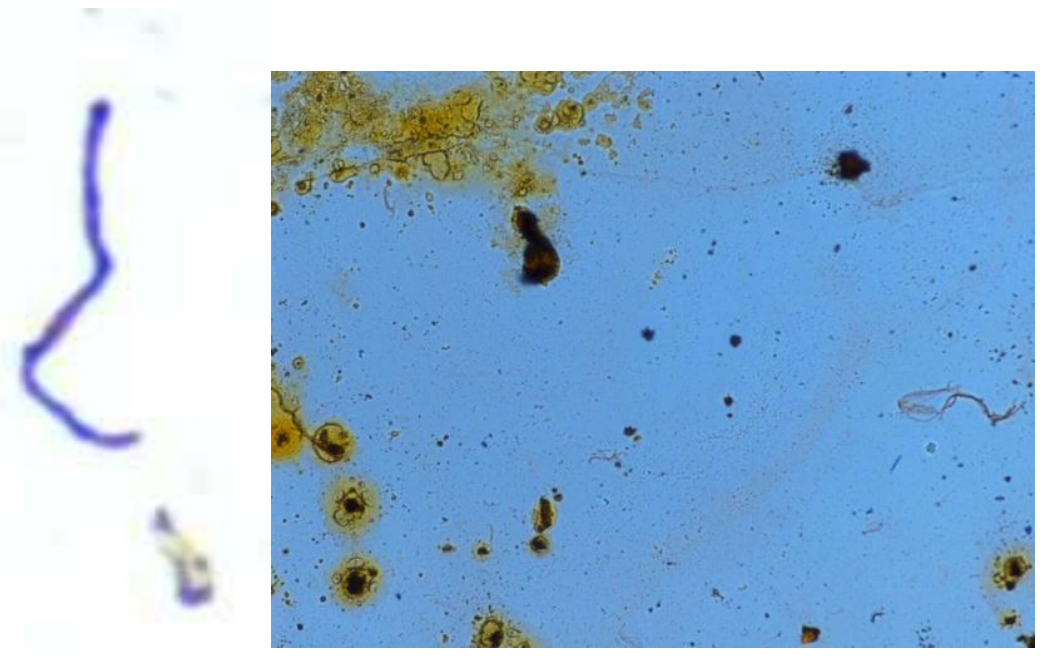

Figure 7:- Testis treated with RNase A : Sperm was alive but the head got separated from the tail and cyst formation is seen.

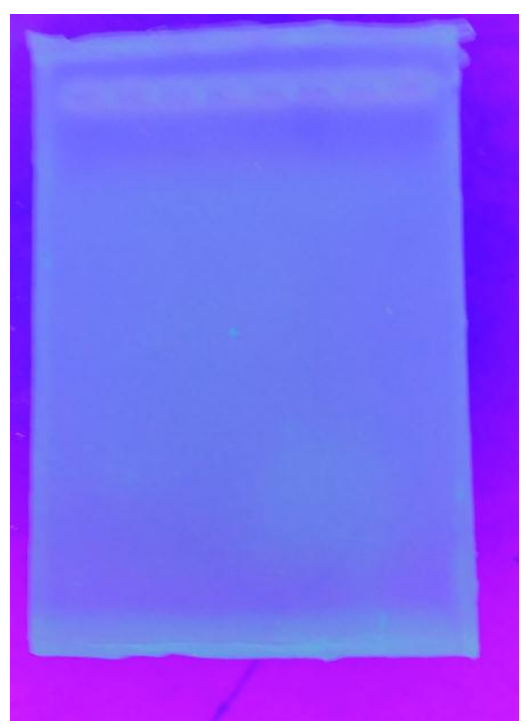

Figure 8:-Agarose gel electrophoresis of treated samples as per the experimental design. The sample was so fragmented so it got mixed up with all the wells.

\section{Materials and Methods:-}

Albino rats were obtained from the Bangalore and maintained in animal house and rat feed was provided once in a day. For this experiment nearly 6 months aged rats was kept as one group and nearly 3 months aged rats as one group. In which one rat from each group was dissected, and testis collected was kept in deep freezer $\left(-20^{0} \mathrm{c}\right)$ for nearly 1 month and minced using scissors in to fine suspension in phosphate buffered saline and same procedure was followed for fresh sample except storing in freezer .

\section{Staining:-}

The protocol was taken from Hammadeh et al ${ }^{[5]}$ with minor modifications which are briefly explained here. 5 microliters of suspension was placed on the glass slide, smear was prepared, air dried and fixed in $3 \%$ glutaraldehyde solution in petriplate and incubating the slides in it for $30 \mathrm{~min}$. The slide was washed twice with PBS PH 7.4 for $5 \mathrm{~min}$ each and was stained with 5\% acidic aniline blue stain (5g aniline blue 4\%glacial acetic acid in double distilled water PH3.5) for 10 min and observed under the compound microscope. 


\section{Experimental design for micronuclei in spems:-}

Rats were dissected and testis was collected and treated with RNaseA alone $100 \mu 1$ from $1 \mathrm{mg} / 1 \mathrm{ml}$ solution, drug alone $100 \mu 1$, both RNaseA and drug metosartan $(25 \mathrm{mg} / 25 \mathrm{ml})$ and control with only water.

\section{Isolation of DNA:(7)}

Testis was homogenized in lysis buffer(50 mMTris-Cl, pH 8.0, $100 \mathrm{mM}$ EDTA, 0.125\% SDS) using mortar and pestle after treating them according to the experimental design and $1 \mathrm{ml}$ of the lysis buffer was added to the supernatant and incubated for 5hrs and phenol: chloroform: isoamylalcohol(25:24:1)to the lysate and centrifuged at $2000 \mathrm{xg}$ for $10 \mathrm{~min}$. The aqueous phase was collected and washed once with phenol chloroform and isoamyl alcohol mixture $1 \mathrm{ml}$ and chloroform $3 \mathrm{ml}$. The aqueous phase was separated and the DNA was precipitated with chilled ethanol and the precipitated DNA was pelleted and stored in deep fridge.

Agarose gel Electrophoresis:-

Agarose gel electrophoresis was performed with isolated DNA samples and visualised under uv trans illuminator

\section{Acridine orange Staining:-}

The separated DNA was mixed with $0.1 \%$ agarose and slide were prepared, allowed to dry and stained with acridine orange and immediately visualised under fluorescent microscope.

\section{Acknowledgements:-}

JRF from the UGC- CSIR is greatly acknowledged.

\section{References:-}

1. Robert love etal; (1959) Activity of Ribonuclease in preparations of testicular and streptococcal hyaluronidase, J histochemcytochem 437-449.

2. AnkurMaheshwarietal; (2008) Pathways involved in testicular germ cell apoptosis induced by $\mathrm{H} 2 \mathrm{O} 2$ in vitro ,870-881.

3. Robert M., etal; (2010) Chromatin condensation a modulates access and binding of nuclear proteins FASEB J. Apr; 24(4): 1066- 10

4. Landré JB etal; (2002) Human endothelial cells selectively express large amounts of pancreatic-type ribonuclease(RNase 1). J Cell Biochem.; 86(3):540-52

5. Mukopadhayayetal (2011) Effect of cryopreservation on sperm chromatin integrity and fertilizing potential in bovine semen Volume 136, Issues 2-3, Pages 114-121.

6. JEFFREY A. NICKERSON etal., (1989 ) Chromatin architecture and nuclear RNA Vol. 86, pp. 177-181, January Cell Biology.

7. John R. Hofstetter, Aiwu Zhang, Aimee R. Mayeda, Tim Guscar, John I. Nurnberger, Jr., and Debomoy K. Lahiri Genomic DNA from Mice: A Comparison of Recovery

8. Methods and Tissue Sources. BIOCHEMICAL AND MOLECULAR MEDICINE 62, 197-202 (1997). 